\title{
Multi-view Dense Match for Forest Area
}

\author{
Wang Tao \\ Guangzhou Institute of Geography, China; \\ wangtao8869@163.com
}

Commission VI, WG VI/4

KEY WORDS: Lidar, dense matching, forest

\begin{abstract}
:
LIDAR(Light Detection And Ranging) is widely used in forestry applications to obtain information about tree density, composition, change, etc. An advantage of LIDAR is its ability to get this information in a 3D structure. However, the density of LIDAR data is low, the acquisition of LIDAR data is often very expensive, and it is difficult to be utilised in small areas. In this article we eavluate different methods by using multi-view to acquire high resolution images of the forest. Using the dense match method a dense point cloud can be generated. Our analysis shows that this method can provide a good alternative to using LIDAR in situations such as these.
\end{abstract}

\section{INTRODUCTION}

Using point data one can extract an object's 3D information and structure. Point matching means matching corresponding points between two or more images of the same scene and this is an important feature of many computer vision and pattern recognition tasks, including object recognition and tracking and 3D scene reconstruction. This makes point data a very important source for data mapping purposes. Because of the importance of point cloud data for many applications LIDAR data is widely used in many projects. As Bartels and Wei comment "is an important modality in terrain and land surveying for many environmental, engineering and civil applications"(Bartels and Wei, 2010). However, when using LIDAR to extract the parameters and characteristics of forest areas there are a number of problems with the approach namely: data resolution, cost, and data processing requirements. it is a good test by using multi-view match to generate dense match cloud point.

The European spatial data research organization started a benchmark on image based DSM generation in feburay 2013. This test is based on two representative image blocks, which were processed by different groups with different software systems.

The interpretation of the benchmark results identified some scenarios that still can cause some problems during image based surface reconstruction. Some solutions showed decreasing accuracies as shadows were cast. In addition, the representative image blocks don't include the forest area (Haala, 2013).

Dense matching methods can be divided into two types: depth map fusion and object patch. One technique used to reduce the search area of matching processes in aerial images is MVLL (Multi-View Local Learning), which defines a vertical line in the object space and calculates the correlation coefficients of the two image matrices according to a point along this line (Zhang, 2005; Waser, 2008; Baltsavias, 2008; Jiang, 2004; Ming, 2009).

Dynamic programming is a method for efficiently solving optimization problems by caching subproblem solutions rather than recomputing them again, dynamic programming was a subglobal matching strategy where one 1D constraint is considered along image rows, the use of those row-wise 1D constraints results in depth maps that show a "streaking effect"(H.Liu, 2011; Y.Liu, 2011; Hirschmuller, 2008; Szeliski, 2011).

Interscanline inconsistencies might be resolved by techniques which use 2D constraints, like graph cut, belief propagation stereo analysis.

SGM algorithm is called semi-global matching. it is published by H.Hirschmuller in 2005. It approximates global optimization by combining multiple $1 \mathrm{D}$ optimization criteria.

Baltsavias and Waser developed a new image matching software package. They demonstrated its application in 3D tree modelling by comparing this to data obtained by the airborne laser. It showed that photogrammetric DSM (Digital Surface Models) can be denser than a DSM generated by LIDAR. Leberl compared point clouds from aerial and street-side LIDAR systems with those created from images. They show that the photogrammetric accuracy compares very well with the LIDAR method. However the key advantage of the photogrammetric approach is that the density of surface points is much higher from the images than from the LIDAR method. The authors conclude that "throughput is commensurate with a fully automated all-digital approach". When image capture has been completed the next step is to manage and process the collection of images.

In this article, we try to evaluate the dense match algorithm in forest area. section 2 show the algorithm of the dense match methods, section 3 shows the test area and results, sections 4 gives the conclusion.

\section{ALGORITHM}




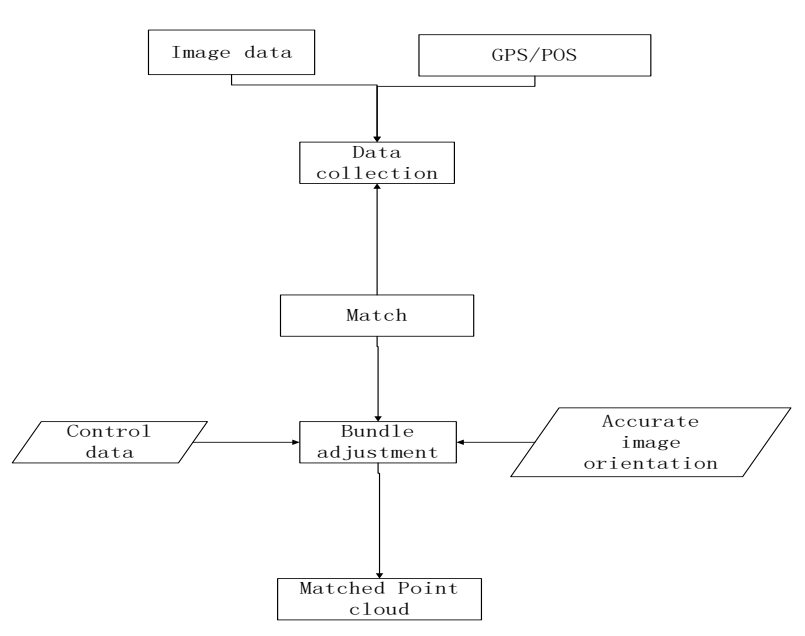

Figure 1 workflow of the processing

Under forest area, it is hard to set control point in the forest. So we get the image orientation parameter by using GPSsupported bundle adjustment. By using multi-view dense match algorithm, we generate dense matched cloud point.

We use SIFT algorithm to generate connection point.

We use calibrated bundle adjustment to calculate the accurate orientation and camera distortion.

$$
\begin{aligned}
& x-x_{0}-\Delta x=-f \frac{a_{1}\left(X-X_{s}\right)+b_{1}\left(Y-Y_{s}\right)+c_{1}\left(Z-Z_{s}\right)}{a_{3}\left(X-X_{s}\right)+b_{3}\left(Y-Y_{s}\right)+c_{3}\left(Z-Z_{s}\right)}=-f \frac{\bar{X}}{\bar{Z}} \\
& y-y_{0}-\Delta y=-f \frac{a_{2}\left(X-X_{s}\right)+b_{2}\left(Y-Y_{s}\right)+c_{2}\left(Z-Z_{s}\right)}{a_{3}\left(X-X_{s}\right)+b_{3}\left(Y-Y_{s}\right)+c_{3}\left(Z-Z_{s}\right)}=-f \frac{\bar{Y}}{\bar{Z}}
\end{aligned}
$$

as to the dense match method, I use three kind of methods, they are PMVS, Pix4d and SURE.

PMVS is a patch based method. this method is implemented as a match, expand, and filter procedure, starting from a sparse set of matched keypoints, and repeatedly expanding these to nearby pixel correspondences before using visibility constraints to filter away false matches (Zhu, 2009; Furukawa, 2008; Furukawa and Jean, 2009; Furukawa et al., 2009b; Shi, Guo and Hu, 2011).

Pix 4d is photogrammetry software for UAV.

We use SURE algorithm to generate the dense matched cloud point. There are three main modules in the algorithm. image rectification module is to generate epipolar images for the matching process. then it is dense stereo matching module. structure computation module is the algorithm for 3D object point triangulation.

The SGM algorithm aims to estimate disparities across stereo pairs such that the global cost function

$$
\begin{aligned}
& E(\mathrm{D})=\sum_{x_{b}}\left(C\left(\mathrm{x}_{b}, \mathrm{D}\left(\mathrm{x}_{b}\right)\right)\right)+ \\
& \sum_{x_{N}} P_{1} T\left[\left\|\mathrm{D}\left(\mathrm{x}_{b}\right)-\mathrm{D}\left(\mathrm{x}_{N}\right)\right\|=1\right]+ \\
& \sum_{x_{N}} P_{2} T\left[\left\|\mathrm{D}\left(\mathrm{x}_{b}\right)-\mathrm{D}\left(\mathrm{x}_{N}\right)\right\|>1\right]
\end{aligned}
$$

is minimized. Thereby $\mathrm{D}$ represents the disparity image holding disparity estimates of all base image pixels $\mathrm{x}_{b} . T$ is an operator evaluating to one if the subsequent condition is true and evaluates to zero else. $\mathrm{X}_{N}$ denote base image pixels in the neighborhood of $\mathrm{x}_{b}$. The global cost function $E$ is composed of a data term and two terms claiming for smooth surfaces. The data term is computed by pixel-wise similarity measures $C\left(\mathrm{x}_{b}, \mathrm{x}_{m}\right)$. The penalty parameters $P_{1}$ and $P_{2}$ control the gain of surface smoothing(Rothermel et al., 2011; Rothermel et al., 2012; Wenzel et al., 2013).

\section{MATERIAL}

\subsection{Study area}

The test site is located at Zhangye, Gansu Province in Western China ( $38^{\circ} 32 \_$N, $100^{\circ} 15_{-}$E). The elevation of the test site is about $2800 \mathrm{~m}$. The forest is a pure spruce (Piceacrassifolia) stand. The forest floor is covered by moss. Dead branches remain on thelower trunks of some trees. A 100 $\mathrm{m} \times 100 \mathrm{~m}$ field plot in relatively flat terrain wasselected for data collection. There were more than 1400 trees with diameter at breastheight $(\mathrm{DBH})$ greater than $2.5 \mathrm{~cm}$ within the plot. Range images used in this study wereacquired on 8 June 2008.

\subsection{LiDAR and aerial imagery}

The LiDAR data and the aerial imagery were collected May, 2008, using a LiteMapper 5600 airborne laser scanner and DigiCAM-H/22 (Digital Camera System 22 megapixels), respectively. The LiDAR was operated at a nominal altitude of $2800 \mathrm{~m}$ above ground level and recorded the first returns as well as the return intensity in a single pass. A $80 \%$ overlap between adjacent strips ensured that density is enough in the surveyed area. The maximum scan angles were $\pm 30^{\circ}$ off nadir and the average sampling space is about $1.6 \mathrm{~m}(0.43 / \mathrm{m} 2$ for whole area, $0.57 / \mathrm{m} 2$ for broadleaf tress and $0.65 / \mathrm{m} 2$ for conifer trees). The nominal accuracy of horizontal (x,y) and vertical (z) is about 0.5 and $0.2 \mathrm{~m}$, respectively. The DigiCAM is a charge coupled device (CCD) camera with 22 mega pixels and each pixel is 9 $\mu \mathrm{m}$ in size. The DigiCAM imagery has a $50 \mathrm{~cm}$ spatial resolution with a $80 \%$ overlap along the flying direction and a $80 \%$ overlap across flight lines.

\subsection{Field data}

The study area is called super area, because area is covered with all kinds of investigation methods. Super study area is $100 \mathrm{~m}$ plus $100 \mathrm{~m}$, the study area is divided into 16 small area, $25 \mathrm{~m}$ plus $25 \mathrm{~m}$. Ground control point were measured by differential GPS. Reference data for tree heights were measured manullay by total station. Terrestrial laser scanner used in this study. Figure 2 shows the image of the whole area. Figure 3 shows the lidar data of test area. 


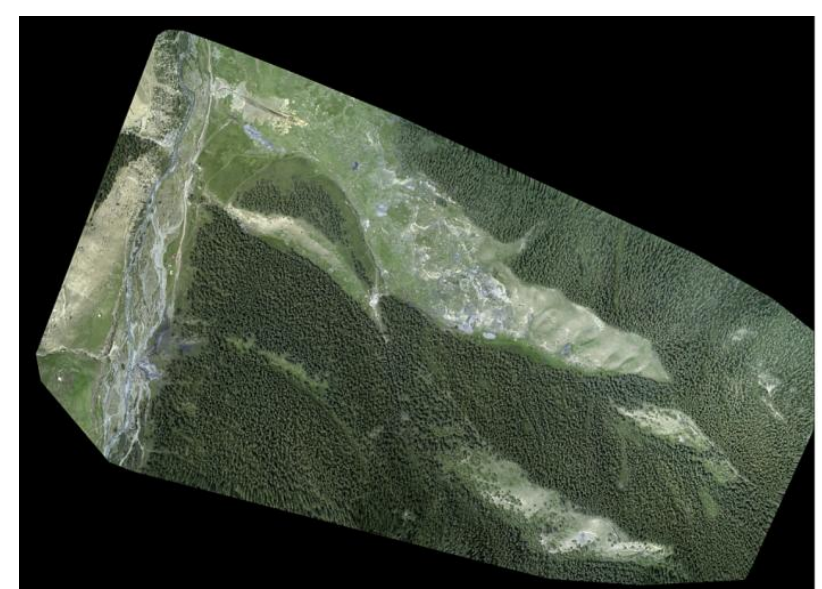

Figure 2. mosaic image of the whole area

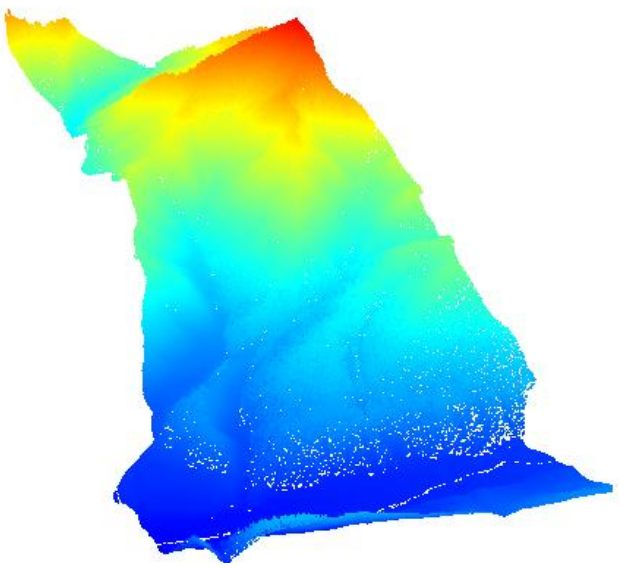

Figure 3. cloud point from lidar

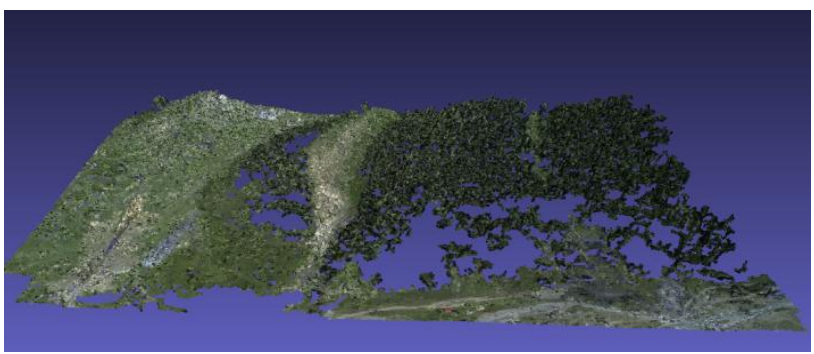

Figure 4.cloud point generated by pmvs

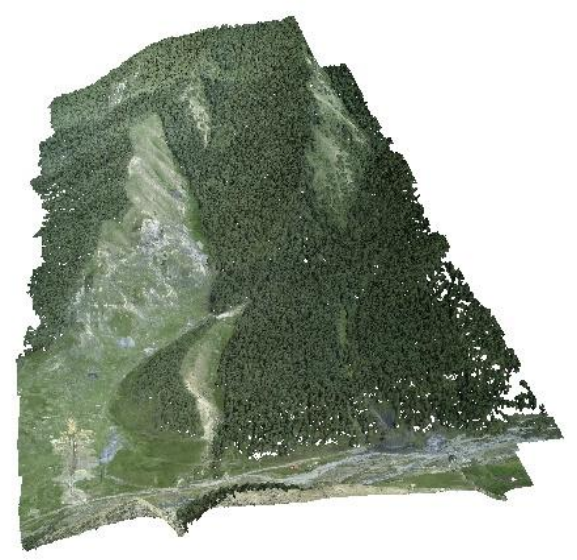

Figure 5 cloud point generated by pix $4 d$

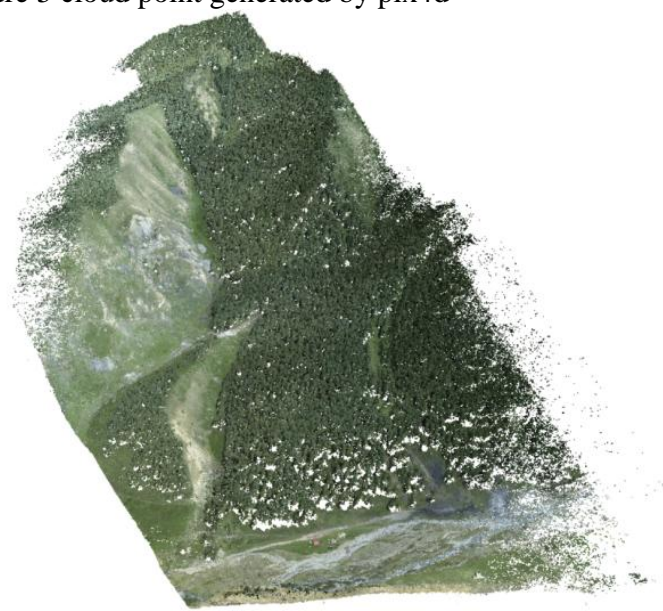

Figure 6 point cloud generated by SURE

PMVS didn't show good result in the test area, so I didn't calculate its density. Density of SURE matched point cloud is 66 points $/ m^{2}$, density of pix 4 d matched point cloud is 7 points/ $m^{2}$, density of the lidar is $3 \sim 4$ points/ $m^{2}$. In the test area, there are many control point in the test area, so we use them to check the accuracy of the dem of the lidar, we use the height of the trees to check the accuracy of the lidar and matched point cloud. Figure 4 shows the DSM generated by the PMVS. Figure 5 shows cloud point generated by Pix 4 d. Figure 6 shows cloud point generated by SURE. The The avgabs (average of the absolute value of the error), rms (route mean square), stddev (standard deviation), and avg (average)of lidar are 8.4986/9.58415/4.45425/8.487. Matched cloud point are $10.5831 / 10.9816 / 2.93263 / 10.5831$.

\section{CONCLUSION}

In this paper we have evaluate three methods for dense point cloud extraction from multi-view imagery for the purposes of forestry analysis. Following from the experimental detail outlined in the previous section we show the point clouds generated for the forest area in Figure 4 in both Figure 5 and Figure 6. Figure 5 shows the point cloud for the entire area while Figure 6 shows the point cloud for one of the local areas with the test mosaic. both methods can generate dense matched point cloud than lidar. SURE's result present the whole structure of the forest area.

The accuracy of DSM should be improved for extraction of tree parameter. I think the dense match algorithm and fusion algorithm are the two important area.

\section{ACKNOWLEDGEMENTS (OPTIONAL)}

Data for this work were provided by the Key Project of Chinese National Programs for Fundamental Research and Development (973 program No. 2007CB714404), the Knowledge Innovation Program of the Chinese Academy of Sciences (kzcx2-YW-313) and the National Natural Science Foundation of China (grant no. 40730525 and 40971203).

\section{REFERENCES}


1.Baltsavias, E., Gruen, A., Eisenbeiss,H., Zhang,L. and Waser, L. T., 2008. "High-quality image matching and automated generation of 3D tree models." International Journal of Remote Sensing 29 (5), 1243-1259.

2. Furukawa Yasutaka, 2008. "High-Fidelity IMAGE-BASED MODELING." Ph D. diss., University of Illinois.

3. Furukawa Yasutaka and Jean Ponce, 2009. "Accurate, Dense, and Robust Multi-View Stereopsis." IEEE Transactions on Pattern Analysis and Machine Intelligence.

4. Furukawa Yasutaka, Brian Curless, Steven M. Seitz, and Richard Szeliski, 2009. "Reconstructing Building Interiors from Images." paper presented at International Conference on Computer Vision.

5. Haala, N., 2013. "The Landscape of Dense Image Matching Algorithms." paper presented at Photogrammetric Week 2013, Wichmann Verlag, Berlin/Offenbach.

6. Hirschmüller, H., 2008. "Stereo Processing by Semiglobal Matching and Mutual Information." IEEE Transactions on Pattern Analysis and Machine Intelligence 30, 328-341.

7. Jiang Wanshou, 2004. "Multiple Aerial Image Matching and Automatic Building Detection." Ph D. diss., University of Wuhan.

8. Leberl F, A. Irschara, T. Pock, P. Meixner, M. Gruber, S. Scholz, and A. Wiechert, 2011. "Point Clouds: Lidar versus 3D Vision." Photogrammetric \& Remote Sensing 76 (10): 11231134.

9. Liu Hongxin, 2011. "Research on algorithm of stereo correspondence based on graph cut." Dissertation. University of Hunan.

10. Liu Yingjie, 2011. "Research on stereo matching algorithm based on dynamic programming and belief propagation." Dissertation. Yanshan University of Yanshan.

11.Ming Yang, 2009. "Research on Key Technologies of Automatic Image Matching for Special Aerial Images.” Ph D. diss., University of Wuhan.

12. Marc Bartels, Hong Wei, Threshold-free object and ground point separation in LIDAR data, Pattern Recognition Letters, Volume 31, Issue 10, July 2010, Pages 1089-1099,

13.Rothermel, M., Wenzel, K., Fritsch, D., Haala, N, 2012. "SURE: Photogrammetric Surface Reconstruction from Imagery." paper presented at Proceedings LC3D Workshop, Berlin. December.

14.Rothermel, M., Haala, N., 2011. "Potential of Dense Matching for the Generation of High Quality Digital Elevation Models.” paper presented In ISPRS Proceedings XXXVII 4W19.

15.Shi Limin, Guo Fusheng, Hu Zhanyi, 2011. "An Improved PMVS through Scene Geometric Information." ACTA AUTUMATICA SINUCA 37 (5): 560-568.

16.Szeliski, Richard, 2011. Computer Vision: Algorithm and Applications. Springer.

17.Waser, L.T., Baltsavias, E., Ecker, K., Eisenbeiss, H., Ginzler, C., Küchler, M., Thee, P. and Zhang, L. 2008. "Highresolution digital surface models (DSMs) for modeling fractional shrub/tree cover in a mire environment." International Journal of Remote Sensing, 29 (5): 1261-1276.

18.Wenzel, K., Rothermel, M., Fritsch, D., and Haala, N, 2013. "Image Acquisition and Model Selection for Multi-View Stereo." paper presented at Int. Arch. Photogramm. Remote Sens. Spatial Inf. Sci., XL-5/W1, 251-258, doi: 10.5194/isprsarchives-XL-5W1-251-2013.

19.Zhang Li, 2005. "Automatic Digital Surface Model (DSM) Generation from Linear Array Images." Ph D. diss., Swiss Federal Institute of Technology Zurich for the degree of Doctor of Technical Sciences.
20.Zhu Qing. 2009. "The Study and Realization of Multi-view Image-based 3D Reconstruction Algorithm." Dissertation., University of Lanzhou. 